Document downloaded from:

http://hdl.handle.net/10251/55526

This paper must be cited as:

Marini, S.; Mattes, M.; Gimeno Martinez, B.; Boria Esbert, VE. (2011). Improved computation of propagation losses in waveguide structures using perturbation of boundary conditions. IEEE Microwave and Wireless Components Letters. 21(11):577-579. doi:10.1109/LMWC.2011.2167135.

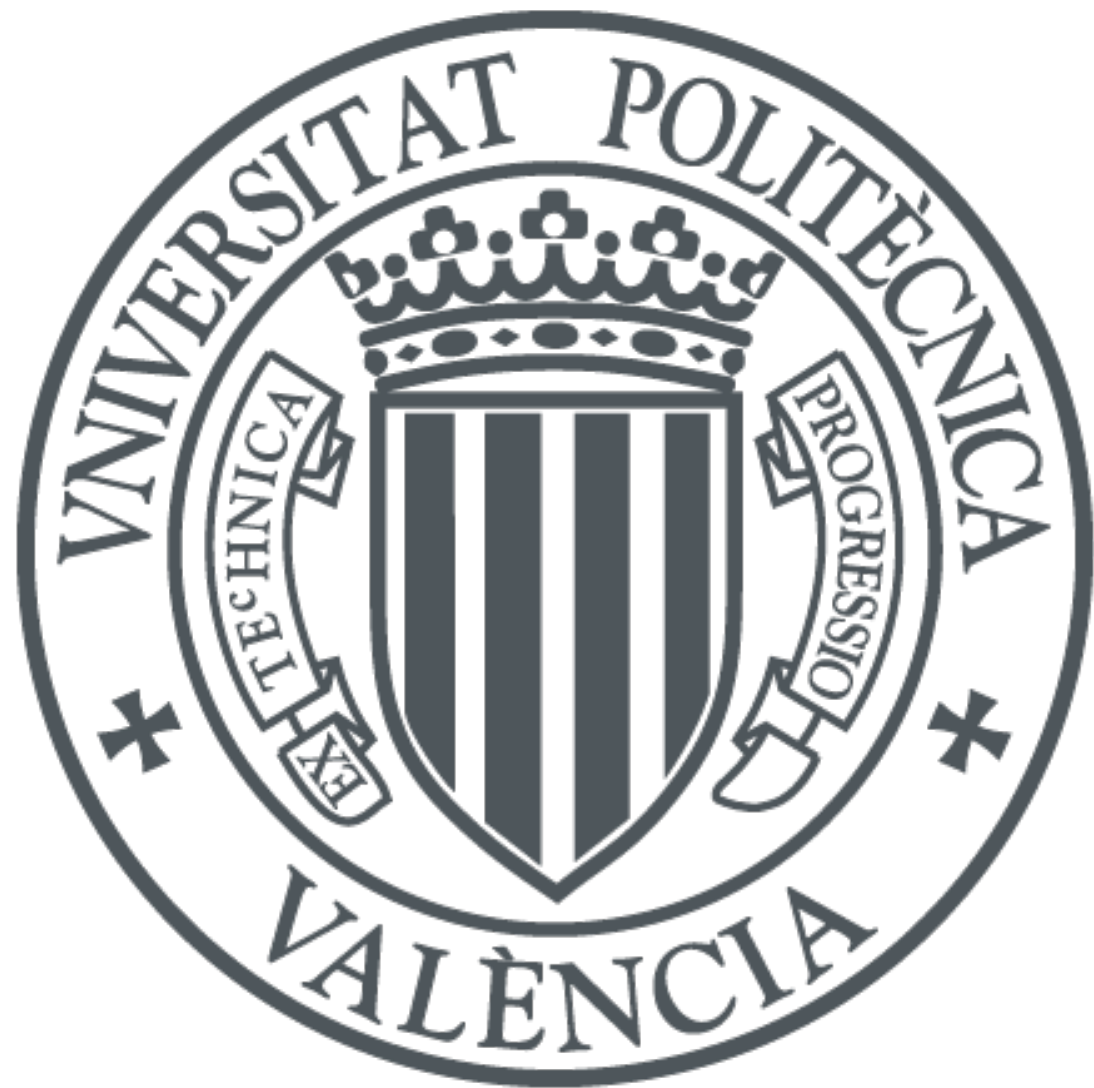

The final publication is available at

http://dx.doi.org/10.1109/LMWC.2011.2167135

Copyright Institute of Electrical and Electronics Engineers (IEEE)

Additional Information

"(C) (C) 20xx IEEE. Personal use of this material is permitted. Permission from IEEE must be obtained for all other uses, in any current or future media, including reprinting/republishing this material for advertising or promotional purposes, creating new collective works, for resale or redistribution to servers or lists, or reuse of any copyrighted component of this work in other works." 


\title{
Improved Computation of Propagation Losses in Waveguide Structures Using Perturbation of Boundary Conditions
}

\author{
Stephan Marini, Member, IEEE, Michael Mattes, Member, IEEE, Benito Gimeno, Member, IEEE and \\ Vicente E. Boria, Senior Member, IEEE
}

\begin{abstract}
In this letter, a method for the improved consideration of propagation losses in metallic waveguide structures is presented. The method relies on the perturbation of the boundary conditions on the metallic walls of the waveguides. Following this advanced technique, we are able to compute a complex modal propagation constant, thus avoiding the drawbacks of the classical power-loss method where losses associated to evanescent modes were not taken into account. A Computer Aided Design (CAD) software package based on such a modal analysis tool has been applied to predict the propagation loss effects in a $\mathrm{Ka}$-Band rectangular waveguide filter.
\end{abstract}

Index Terms-Losses, Waveguide components, Dissipative filters, Complex propagation constant, Perturbation method.

\section{INTRODUCTION}

$\mathbf{M}$ OST of present and future wireless and/or space communication systems are operating at higher frequencies, such as microwave and millimeter-wave bands. At such higher frequencies ohmic losses, though they can be reduced due to silver- or gold-plating, are becoming more and more relevant for the design of passive waveguide components, such as filters and multiplexers used in satellite payloads. The finite values for the material's conductivity degrade the behavior of the passive devices, increase the insertion loss levels and even alter the frequency response. This means that, for design purposes, the conventional approach of neglecting losses or considering them as a small-effect phenomenon is no longer valid. As a consequence, modern CAD tools are requesting the inclusion of ohmic losses, due to imperfect conducting materials, for an accurate full-wave response prediction of passive microwave and millimeter-wave components [1]-[3].

Ohmic losses in microwave waveguides and cavities have been under consideration in the technical literature during the past decades. Propagation losses are often modelled using the

This work has been supported by Ministerio de Educación y Ciencia, Spanish Government, under the Grant JC2009-0221 and the coordinated R\&D project TEC2010-21520-C04, and by University of Alicante under the project GRE10-22.

S. Marini is with the Departamento de Física, Ingeniería de Sistemas y Teoría de la Señal, Instituto Universitario de Física Aplicada a las Ciencias y las Tecnologías, Universidad de Alicante, Spain (e-mail: smarini@dfists.ua.es).

M. Mattes is with the Laboratory of Electromagnetics and Acoustics, Ecole Polytechnique Fédérale de Lausanne (EPFL), Switzerland.

B. Gimeno is with the Departamento de Física Aplicada, Instituto de Ciencia de los Materiales, Universidad de Valencia, Spain.

V.E. Boria is with the Departamento de Comunicaciones, Instituto de Telecomunicaciones y Aplicaciones Multimedia, Universidad Politécnica de Valencia, Spain. perturbative power-loss method [4]. A very early contribution based on this method can be found in [5], where degenerated modes in lossy waveguides and cavities are treated. In [6] a complex power technique and mode-matching procedure have been employed for the analysis of waveguide junctions characterized by the Generalized Scattering Matrix (GSM). More recently, the rigorous consideration of the losses due to the transversal metallic wall of a junction between two rectangular waveguides with different cross sections has been included in [7] and [8].

In this letter, we present an enhanced method for the computation of propagation losses in rectangular waveguide structures, which can be easily integrated within CAD tools for design purposes. This new method, based on the perturbation of boundary conditions, allows to overcome all the drawbacks of the classical power-loss method. It is valid assuming that the metallic walls are good conductors $\left(\sigma>10^{4} \mathrm{~S} / \mathrm{m}\right)$, so the cross-coupling between the modal powers of degenerated modes is negligible. In order to analyze realistic metalbased passive structures, the theory developed has been easily combined with the integral-equation analysis technique (IE) described in [9]. Proceeding in this way, a CAD software package based on such a modal analysis tool has been applied to predict the propagation loss effects in rectangular waveguide filters.

\section{THEORY}

From now and on, and with the aim of simplifying the notation of this section, a zero subscript will be only used to denote the electromagnetic fields for perfectly conducting walls. Note that the time factor $e^{j \omega t}$ is considered and omitted throughout this paper. To study the changes produced by a finite conductivity, we have followed the technique proposed in [10], [11], where only TM modes are treated in detail. In this contribution, we also extend it to the TE case. The formulation starts with the assumption that a small tangential electric field does exist for a good conductor, which is given by the Leontovich condition [10]

$$
\mathbf{E} \simeq Z_{s}\left(\hat{n} \times \mathbf{H}_{\mathbf{0} \|}\right)=\frac{(1+j)}{\sigma \delta}\left(\hat{n} \times \mathbf{H}_{\mathbf{0} \|}\right)
$$

where $\hat{n}$ is the unit normal vector outward from the conductor, $Z_{s}=(1+j) /(\sigma \delta)$ is the surface impedance, $\sigma$ is the metallic conductivity, $\delta=\sqrt{2 /\left(\mu_{c} \omega \sigma\right)}$ is the skin depth, $\mu_{c}$ being the conductor magnetic permeability. 
For large conductivity values, the classical perturbative power-loss method assumes an exponential amplitude attenuation only for the excited propagative modes [4], [10], thus neglecting the ohmic losses related to the evanescent modes. Moreover, this attenuation constant tends to infinity at the cutoff modal frequency. All these drawbacks can be overcome using the perturbation of boundary conditions technique [10], [11], which is based on the calculation of a complex propagation wavenumber $k_{z}=\beta-j \alpha$. In this method both attenuation and phase constants take finite and positive real values, even at the modal cut-off frequency. In this way, losses associated with the propagative and evanescent modes are rigorously accounted for.

Let us consider a waveguide with any arbitrary cross-section which is homogeneously filled by a lossless medium with constants $\mu$ and $\epsilon$. We start considering the lossless TM problem, described as follows

$$
\left(\nabla_{t}^{2}+k_{c 0}^{2}\right) E_{z 0}=\left.0 \quad E_{z 0}\right|_{\partial S}=0
$$

where $k_{c 0}=\omega_{c 0}^{2} \mu \epsilon$ is the modal cut-off wavenumber. It is well known that the transverse magnetic field can be expressed in terms of the longitudinal electric field as shown next [10]:

$\mathbf{H}_{\mathbf{t o}}=\frac{\omega \epsilon}{k_{z 0}} \hat{z} \times\left(\frac{-j k_{z 0}}{k_{c 0}^{2}} \nabla_{t} E_{z 0}\right)=\frac{-j \omega \epsilon}{k_{c 0}^{2}}\left[\hat{l} \frac{\partial E_{z 0}}{\partial n}-\hat{n} \frac{\partial E_{z 0}}{\partial l}\right]$

where $k_{z 0}^{2}=\omega^{2} \mu \epsilon-k_{c 0}^{2}$, and $\hat{l}$ is a unit tangential vector to the conductor surface. Thus, for a finite but large conductivity value, a non-zero axial electric field component appears, which can be easily calculated combining (1) and (3), obtaining

$$
\left.\left.E_{z}\right|_{\partial S} \simeq \frac{(1-j)}{2} \frac{\mu_{c}}{\mu} \delta\left(\frac{\omega}{\omega_{c 0}}\right)^{2} \frac{\partial E_{z 0}}{\partial n}\right|_{\partial S} .
$$

So, the proposed technique for considering losses is described by the following equivalent perturbed TM problem:

$$
\left(\nabla_{t}^{2}+k_{c}^{2}\right) E_{z}=0 ;\left.\left.\quad E_{z}\right|_{\partial S} \simeq f_{T M} \frac{\partial E_{z 0}}{\partial n}\right|_{\partial S}
$$

with $f_{T M}=\frac{(1-j)}{2} \frac{\mu_{c}}{\mu}\left(\frac{\omega}{\omega_{c 0}}\right)^{2} \delta$, and $k_{c}$ the complex cut-off wavenumber.

In order to obtain the complex propagation wavenumber, the Green theorem in two dimensions is employed [10]. Then, considering that $f_{T M}$ is assumed to be a parameter with a small value $\left(f_{T M}<<1 \mathrm{~mm}\right)$, it is possible to approximate $E_{z}$ by its unperturbed value $E_{z 0}$, thus obtaining

$$
k_{z}^{2}-k_{z 0}^{2} \simeq f_{T M} \frac{\oint_{\partial S}\left|\frac{\partial E_{z 0}}{\partial n}\right|^{2} d l}{\int_{s}\left|E_{z 0}\right|^{2} d S}
$$

where $S$ is the cross-sectional surface and $\partial S$ is the boundary of the waveguide. Introducing the following parameter

$$
\xi_{T M}=\frac{f_{T M}}{2(1-j) k_{z 0}} \frac{\oint_{\partial S}\left|\frac{\partial E_{z 0}}{\partial n}\right|^{2} d l}{\int_{s}\left|E_{z 0}\right|^{2} d S}
$$

yields the new complex propagation wavenumber

$k_{z}=\beta-j \alpha=\sqrt{k_{z 0}\left(k_{z 0}+2 \xi_{n}\right)-j 2 \xi_{n} k_{z 0}} \quad n=T M, T E$.

which is also valid for TE modes as will be shortly shown.
The TE modes for the lossless problem are obtained solving the following eigenvalue problem

$$
\left(\nabla_{t}^{2}+k_{c 0}^{2}\right) H_{z 0}=0 ;\left.\quad \frac{\partial H_{z 0}}{\partial n}\right|_{\partial S}=0 .
$$

For metallic walls with a large and finite conductivity value, a non-zero electric tangential field appears on the surface (1),

$$
\left.\left.\mathbf{E}_{\|}\right|_{\partial S} \simeq\left(Z_{s} \frac{-j k_{z}}{k_{c 0}^{2}} \frac{\partial H_{z 0}}{\partial l}\right)\right|_{\partial S} \hat{z}-\left.Z_{s} H_{z 0}\right|_{\partial S} \hat{l} .
$$

Then, the normal component of the magnetic field associated to the axial electric field can be calculated by means of the Maxwell equations [10], obtaining

$$
\left.\left.H_{n}\right|_{\partial S} \simeq \frac{j}{\mu_{c} \omega} \frac{\partial}{\partial l}\left(Z_{s} \frac{-j k_{z}}{k_{c 0}^{2}} \frac{\partial H_{z 0}}{\partial l}\right)\right|_{\partial S}=\left.\frac{Z_{s} k_{z}}{\mu_{c} \omega k_{c 0}^{2}} \frac{\partial^{2} H_{z 0}}{\partial l^{2}}\right|_{\partial S}
$$

By defining $f_{T E}=-(1-j) \frac{\delta}{2}$, the corresponding perturbed TE problem, equivalent to (9), is given by

$$
\begin{gathered}
\left(\nabla_{t}^{2}+k_{c}^{2}\right) H_{z}=0 \\
\left.\left.\frac{\partial H_{z}}{\partial n}\right|_{\partial S} \simeq f_{T E}\left(\omega^{2} \mu \epsilon \frac{\mu_{c}}{\mu} H_{z 0}-\frac{k_{z 0}^{2}}{k_{c 0}^{2}} \frac{\partial^{2} H_{z 0}}{\partial l^{2}}\right)\right|_{\partial S}
\end{gathered}
$$

The last step in the derivation is to use the Green theorem in two dimensions to obtain the following parameter

$$
\begin{aligned}
\xi_{T E}= & \frac{-f_{T E}}{2(1-j) k_{z 0}} \frac{\oint_{\partial S}\left(\omega^{2} \mu \epsilon \frac{\mu_{c}}{\mu}\left|H_{z 0}\right|^{2}-\frac{k_{z 0}^{2}}{k_{c 0}^{2}} \frac{\partial^{2} H_{z 0}}{\partial l^{2}}\right) d l}{\int_{s}\left|H_{z 0}\right|^{2} d S}= \\
& \frac{\delta}{4 k_{z 0}} \frac{\oint_{\partial S}\left(\omega^{2} \mu \epsilon \frac{\mu_{c}}{\mu}\left|H_{z 0}\right|^{2}+\frac{k_{z 0}^{2}}{k_{c 0}^{2}}\left|\frac{\partial H_{z 0}}{\partial l}\right|^{2}\right) d l}{\int_{s}\left|H_{z 0}\right|^{2} d S} .
\end{aligned}
$$

Finally, the TE complex propagation wavenumber can be obtained using again the expression (8).

\section{RESULTS}

We proceed to study the accuracy and efficiency of the proposed analysis technique through several examples. Note that in all the presented figures we indicate with method 1 the results obtained by the classical power-loss method, and with method 2 the results obtained by the proposed enhanced technique. First of all, we have performed the modal analysis of a WR-28 rectangular waveguide ( $a=7.112 \mathrm{~mm}$ and $b=3.556 \mathrm{~mm}$ ) with a metal conductivity of $\sigma=5.8 \cdot 10^{7} \mathrm{~S} / \mathrm{m}$. Fig. 1(a) shows in a semi-logarithmic scale the attenuation constant of the first four waveguide modes as a function of the frequency computed using method 1, method 2 and the commercial software HFSS $^{1}$. It should be remarked that method 2 does not fail at the cut-off frequency in contrast with the classical power-loss method. In Fig. 1(b) we also present the modal phase constant for the same modes computed with method 2 and HFSS results. A very good agreement between our simulations and HFSS data has been obtained for conductivity values greater than $\sigma>10^{4} \mathrm{~S} / \mathrm{m}$.

As a second example, we consider a simple WR-28 cavity structure coupled to input/output WR-28 waveguides by two

\footnotetext{
${ }^{1}$ http://www.ansoft.com/products/hf/hfss12/
} 


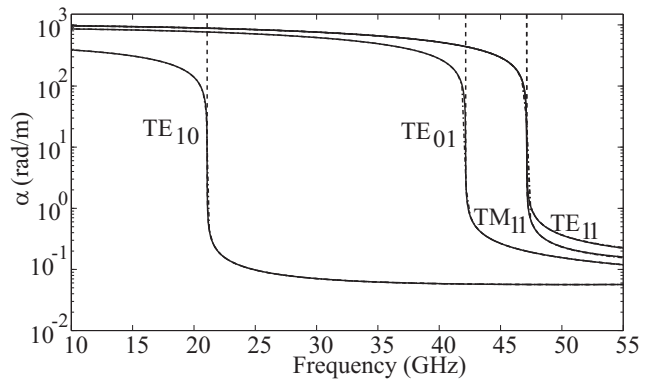

(a)

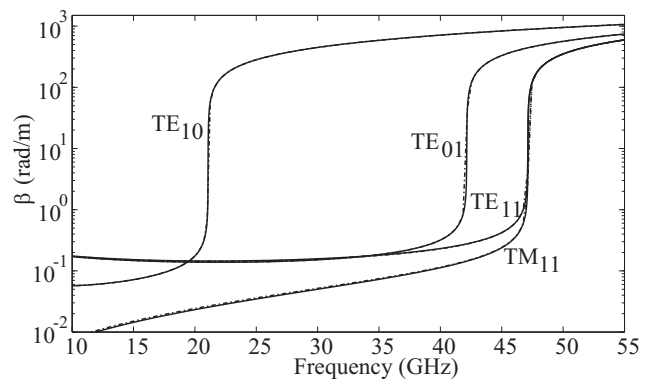

(b)

Fig. 1. Attenuation (a) and phase constants (b) for the first four WR-28 rectangular modes computed with method 1 (dashed lines only in (a)), method 2 (solid lines) and HFSS software (dashdot lines).

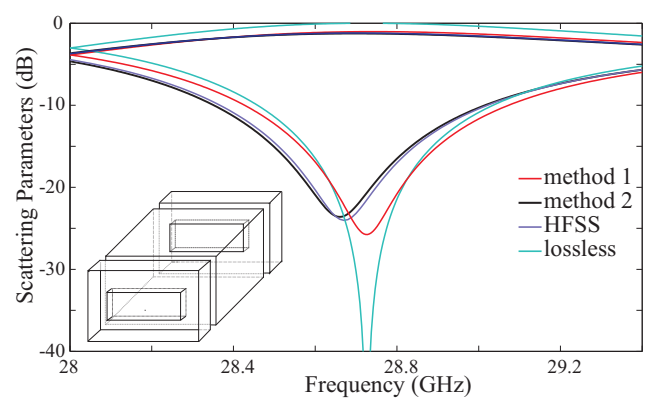

Fig. 2. Scattering parameters of a simple cavity structure coupled to input/output WR-28 waveguides by two irises. Length of WR-28 waveguide cavity is $12 \mathrm{~mm}$, irises dimensions are: $a=4.9 \mathrm{~mm}, b=2.845 \mathrm{~mm}$ and $l=2 \mathrm{~mm}$.

centered irises: we have assumed a low finite conductivity value of $\sigma=10^{5} \mathrm{~S} / \mathrm{m}$. In Fig. 2 the simulated reflection and transmission coefficients of this coupled resonator are compared with the numerical data provided also by HFSS (a maximum value of 0.001 for the S-parameter variation has been used as a convergence criterion). It can be clearly noticed that, due to the low finite conductivity value considered in this case, the change in the propagation constant results in a phase shift (see magnitude of $S_{11}$ ), generating a variation of the resonance frequency of the structure. This last shift is not predicted by the classical method 1 .

Finally, making use of our enhanced CAD software package we have designed an inductive 4-pole rectangular filter in standard WR-28 rectangular waveguides $\left(\sigma=3.54 \cdot 10^{7} \mathrm{~S} / \mathrm{m}\right)$ operating at $40 \mathrm{GHz}$ with a passband of $0.2 \%$. The complete reflection and transmission response of this inductive 4-pole filter is shown in Fig. 3. It can be observed that the deviation

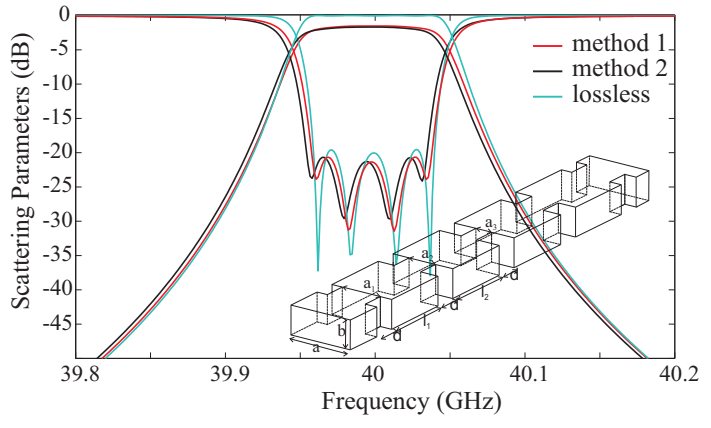

Fig. 3. Magnitude of the scattering parameters of a 4-pole inductive rectangular filter in standard WR-28 waveguide. Coupling irises dimensions: $a_{1}=2.207 \mathrm{~mm}, a_{2}=1.164 \mathrm{~mm}, a_{3}=1.105 \mathrm{~mm}$, and length $d=1 \mathrm{~mm}$. Cavity lengths: $l_{1}=4.072 \mathrm{~mm}, l_{2}=4.286 \mathrm{~mm}$.

between the response obtained with the proposed method and method 1 is around $12 \mathrm{MHz}$, i. e., $3 \%$ of the filter response bandwidth which can be critical for narrow-band applications.

\section{CONCLUSIONS}

In this letter, an improved method for the computation of propagation losses in metallic waveguide structures is presented. Following this technique, the propagation loss effects on the waveguide contour are accurately predicted using a perturbation method. With this approach the drawbacks of the power-loss method can be overcome, and losses associated with evanescent modes can be taken into account, too. Furthermore, the proposed enhanced method is able to predict a phase shift of the electrical filter response, which can not be predicted by the classical power-loss method.

\section{REFERENCES}

[1] I. Hunter, L. Bilonet, B. Jarry and P. Guillon, "Microwave filterapplications and technology ," IEEE Trans. Microwave Theory Tech., vol. 50, no. 3, pp. 794-805, Mar. 2002.

[2] V. Miraftab and M. Yu, "Generalized lossy microwave filter coupling matrix syntesis and design using mixed technologies," IEEE Trans. Microwave Theory Tech., vol. 56, no. 12, pp. 3016-3027, Dec. 2008.

[3] M. Oldoni, G. Macchiarella, G. G. Gentili and C. Ernst, "A new approach to the synthesis of microwave lossy filters ," IEEE Trans. Microwave Theory Tech., vol. 58, no. 5, pp. 1222-1229, May 2010.

[4] R. E. Collin, Field Theory of Guided Waves. McGraw-Hill, 1960, ch. 5, pp. 182-183.

[5] J. Justincic, "A general power loss method for attenuation of cavities and waveguides," IEEE Trans. Microwave Theory Tech., vol. 11, no. 1, pp. 83-87, Jan. 1963.

[6] J. Wade, and R. McPhie, "Conservation of complex power technique for waveguide junctions with finite wall conductivity," IEEE Trans. Microwave Theory Tech., vol. 38, no. 4, pp. 373-378, Apr. 1990.

[7] A. Melloni and G. Gentili, "Modellization of losses in $\mathrm{TE}_{011}$ mode waveguide bandpass filter," IEEE Trans. Microwave Theory Tech., vol. 43, no. 11, pp. 2642-2644, Nov. 1995.

[8] J. Hueso, S. Cogollos, B. Gimeno, V.E. Boria, A. Vidal, M. Taroncher, H. Esteban, and M. Guglielmi, "Accurate consideration of metal losses in planar waveguide junctions using an efficient integral equation technique," IEEE MTT-S Int. Microwave Symp. Dig., pp. 1411-1414, June 2004.

[9] G. Gerini, M. Guglielmi, and G. Lastoria, "Efficient integral equation formulations for admittance or impedance representation of planar waveguide junctions," IEEE MTT-S Int. Microwave Symp. Dig., vol. 3, pp. 1747-1750, June 1998.

[10] J. D. Jackson, Classical Electrodynamics. John Wiley and Sons, 2001.

[11] W. Wilcox, Macroscopic Electrodynamics. Baylor University, 2005, part II, ch. 9. 